\title{
A NOTE ON SOME PECULIAR NONLINEAR EXTREMAL PHENOMENA OF THE CHEBYSHEV POLYNOMIALS
}

\author{
by HOLGER DETTE*
}

(Received 29th November 1993)

\begin{abstract}
We consider the problem of maximizing the sum of squares of the leading coefficients of polynomials $P_{i_{1}}(x), \ldots, P_{i_{m}}(x)$ (where $P_{j}(x)$ is a polynomial of degree $j$ ) under the restriction that the sup-norm of $\sum_{j=1}^{m} P_{i}^{2}(x)$ is bounded on the interval $[-b, b](b>0)$. A complete solution of the problem is presented using duality theory of convex analysis and the theory of canonical moments. It turns out, that contrary to many other extremal problems the structure of the solution will depend heavily on the size of the interval $[-b, b]$.
\end{abstract}

1991 Mathematics subject classification: 33C45.

\section{Introduction}

Let $\mathbb{P}_{j}$ denote the set of all polynomials of degree $j, I=\left\{i_{1}, \ldots, i_{m}\right\}$ denote a subset of $\{1, \ldots, n\}$ containing $n$ (i.e. $n \in I, i_{l} \neq i_{k}$ if $k \neq l$ ) and define

$$
P_{I}:=\left\{\left(P_{j}\right)_{j \in I} \mid P_{j} \in \mathbb{P}_{j}, j \in I, \sup _{x \in I-b, b]} \sum_{j \in I} P_{j}^{2}(x) \leqq 1\right\}
$$

as the set of all polynomials of degree $i_{1}, \ldots, i_{m}$ such that the sup-norm of the sum of squares is bounded by 1 on the interval $[-b, b]$. In the following $m_{l}\left(P_{l}\right)$ denotes the leading coefficient of the polynomial $P_{l} \in \mathbb{P}_{l}$ and we are interested in the nonlinear extremal problem

$$
\max \left\{\sum_{l \in I} m_{l}^{2}\left(P_{l}\right) \mid\left(P_{l}\right)_{l \in I} \in P_{l}\right\}
$$

For $I=\{n\}\left(\mathscr{P}_{I}\right)$ yields the well known extremal property of the Chebyshev polynomials of the first kind $T_{n}\left(\frac{x}{b}\right)$ (see e.g. Achieser [1], Natanson [7] or Rivlin [10]). Similar problems were investigated by Dette [4] who considered the maximization of a weighted product of the squared leading coefficients of the polynomials $P_{l}(x)$. All extremal problems in these references satisfy a so called "invariance property" which means that if a solution on one interval, say $[-1,1]$, has been determined, then the solution on another interval can easily be obtained by a linear transformation from the "optimal" polynomials on the interval $[-1,1]$.

* Research supported in part by the Deutsche Forschungsgemeinschaft. 
In this note we will present a complete solution of the (nonlinear) extremal problem $\left(\mathscr{P}_{1}\right)$. It will turn out that the above invariance property is not true any longer for the problem $\left(\mathscr{P}_{I}\right)$ if $m \geqq 2$. While for sufficiently small $b>0$ the Chebyshev polynomial (of the first kind) on the interval $[-b, b]$ of degree $\max _{j=1}^{m} i_{j}$ is a solution of $\left(\mathscr{P}_{1}\right)$ (all other polynomials are vanishing) this is not true any longer for large $b$. Here the structure of the extremal solution depends heavily on the size of the interval $[-b, b]$.

In Section 2 the problem $\left(\mathscr{P}_{l}\right)$ is solved by an application of some results in convex analysis (see Pukelsheim [9]) and the theory of canonical moments (see Studden [12]). It turns out that the problem $\left(\mathscr{P}_{I}\right)$ is dual to a maximization problem of a concave function defined on the set of all probability measures on the interval $[-b, b]$. This problem appears in the theory of optimal experimental design in mathematical statistics (see Dette [3]). While from a statistical point of view the support points and weights of the optimal measure are the main interest it is shown in this paper that the orthogonal polynomials with respect to this measure form essentially the solution of the extremal problem $\left(\mathscr{P}_{I}\right)$. Section 3 deals with some special cases for the set $I$, namely $I=\{1, \ldots, n\}$ and $I=\{n-1, n\}$ and some explicit examples. Finally, in Section 4 , similar problems are investigated which generalize the extremal properties of the Chebyshev polynomials of the second kind.

\section{The solution of $\left(\mathscr{P}_{I}\right)$}

Throughout this paper $\xi$ is a probability measure on the interval $[-b, b]$ and the corresponding orthogonal polynomials with leading coefficient 1 will be denoted by $P_{j}(x, \xi)$ and their (squared) $L_{2}$-norm by $k_{j}(\xi)=\int_{-b}^{b} P_{j}^{2}(x, \xi) d \xi(x)$. The main step for solving the extremal problem $\left(\mathscr{P}_{I}\right)$ is the following duality which is proved in the appendix.

Theorem 2.1. Let $\Xi:=\left\{\xi \mid k_{n}(\xi)>0\right\}$ and $n \in I$, then

$$
\max \left\{\sum_{l \in I} m_{l}^{2}\left(P_{l}\right) \mid\left(P_{j}\right)_{j \in I} \in P_{I}\right\}=\min _{\xi} \max \left\{k_{j}^{-1}(\xi) \mid j \in I\right\}
$$

Moreover, if $\xi^{*}$ is a solution of the problem $\left(\mathscr{D}_{I}\right)$ and

$$
\mathscr{M}\left(\xi^{*}\right)=\left\{j \in I \mid k_{j}\left(\xi^{*}\right)=\min _{i \in I} k_{i}\left(\zeta^{*}\right)\right\}
$$

then $\left\{\sqrt{\alpha_{j} / k_{j}\left(\xi^{*}\right)} P_{j}\left(x, \xi^{*}\right)\right\}_{j \in I}$ is a solution of $\left(\mathscr{P}_{1}\right)$ where $P_{j}\left(x, \xi^{*}\right)$ is the $j$ th monic orthogonal polynomial with respect to the measure $d \xi^{*}(x)$ and the $\alpha_{j}$ are (arbitrary) nonnegative numbers with sum 1 satisfying

$$
\begin{gathered}
\alpha_{j}=0 \text { if } j \in I \backslash \mathscr{M}\left(\xi^{*}\right) \\
\sum_{j \in I} \alpha_{j} k_{j}^{-1}\left(\xi^{*}\right) P_{j}^{2}\left(x, \xi^{*}\right) \leqq 1 \quad \text { for all } x \in[-b, b]
\end{gathered}
$$


The dual problem $\left(\mathscr{D}_{I}\right)$ appears in the theory of optimal experimental design in mathematical statistics and has been solved in the special case $I=\{1, \ldots, n\}$ (see Dette [3]). While statisticians are mainly interested in the support points and weights of the solution $\xi^{*}$ of $\left(\mathscr{D}_{I}\right)$ (they give essentially the points where observations have to be taken in a polynomial regression) Theorem 2.1 shows that the orthogonal polynomials with respect to the measure $d \xi^{*}(x)$ are needed for the solution of the primal problem $\left(\mathscr{P}_{1}\right)$. In order to determine these polynomials (and to solve the dual problem $\left(\mathscr{D}_{I}\right)$ ) some basic facts about canonical moments of probability measures on the interval $[-b, b]$ are needed. The Stieltjes transform with corresponding continued fraction expansions of such a measure $\xi$ is given by

$$
\begin{aligned}
\int_{-b}^{b} \frac{d \xi(x)}{z-x} & =\frac{1 \mid}{\mid z+b}-\frac{2 b \zeta_{1} \mid}{\mid 1}-\frac{2 b \zeta_{2} \mid}{\mid z+b}-\frac{2 b \zeta_{3} \mid}{\mid 1}-\frac{2 b \zeta_{4} \mid}{\mid z+b}-\ldots \\
& =\frac{1}{\mid z+b\left(1-2 \zeta_{1}\right)}-\frac{(2 b)^{2} \zeta_{1} \zeta_{2}}{\mid z+b\left(1-2 \zeta_{2}-2 \zeta_{3}\right)}-\frac{(2 b)^{2} \zeta_{3} \zeta_{4}}{\mid z+b\left(1-2 \zeta_{4}-2 \zeta_{5}\right)}-\ldots
\end{aligned}
$$

where $\zeta_{1}=p_{1}, \zeta_{j}=q_{j-1} p_{j}(j \geqq 2), q_{j}=1-p_{j}(j \geqq 1)$ and $0 \leqq p_{j} \leqq 1$ (see e.g. Lau and Studden [6]). The quantities $p_{j}$ are called the canonical moments of $\xi$. Note that $p_{j+1}$ is undefined whenever $p_{j} \in\{0,1\}$ because in this case the continued fraction terminates. It is well known that the polynomial in the denominator of the $n$th convergent is the $n$th monic orthogonal polynomial with respect to the measure $d \xi(x)$ and that this polynomial is given by the continuant (see Perron, [8, Bd. I, p. 9])

$$
\begin{aligned}
& P_{n}(x, \xi)= \\
& \quad K\left(\begin{array}{crrr}
-(2 b)^{2} \zeta_{1} \zeta_{2} & -(2 b)^{2} \zeta_{3} \zeta_{4} & \ldots & -(2 b)^{2} \zeta_{2 n-3} \zeta_{2 n-2} \\
x+b\left(1-2 \zeta_{1}\right) & x+b\left(1-2 \zeta_{2}-2 \zeta_{3}\right) & \ldots & x+b\left(1-2 \zeta_{2 n-2}-2 \zeta_{2 n-1}\right)
\end{array}\right)
\end{aligned}
$$

and has $L_{2}$-norm

$$
k_{n}(\xi)=\int_{-b}^{b} P_{n}^{2}(x, \xi) d \xi(x)=(2 b)^{2 n} \prod_{j=1}^{n} \zeta_{2 j-1} \zeta_{2 j}
$$

(see Chihara [2] or Wall [13]). The following theorem determines the canonical moments of the solution of the dual problem $\left(\mathscr{D}_{I}\right)$.

Theorem 2.2. The solution $\xi^{*}$ of the dual problem $\left(\mathscr{D}_{I}\right)$ is uniquely determined by its canonical moments $p_{2 j-1}^{*}=\frac{1}{2}(j=1, \ldots, n), p_{2 n}^{*}=1$ and

$$
p_{2(n-j)}^{*}=\max \left\{z_{n-j}\left[1-b^{-2 j} \prod_{i=n-j+1}^{n-1}\left(q_{2 i}^{*} p_{2 i}^{*}\right)^{-1}\right], \frac{1}{2}\right\} \quad j=n-1, \ldots, 1
$$


where $z_{n-j}$ is 1 or 0 according to $n-j \in I$ or $n-j \notin I$.

Proof. In the special case $I=\{1, \ldots, n\}$ a proof of Theorem 2.2 can be found in Dette [3], which can be generalized to arbitrary index sets. For the sake of completeness we provide a different proof in this paper, which is directly based on the duality result of Theorem 2.1 and uses some identities for orthogonal polynomials on compact intervals. Let $\gamma_{n-j}=1-b^{-2 j} \prod_{i=n-j+1}^{n-1}\left(q_{2 i}^{*} p_{2 i}^{*}\right)^{-1}\left(\gamma_{n}=1\right)$, then it is easy to see (observing (2.5) and (2.6)) that $\gamma_{n-j} \geqq \frac{1}{2}$ if and only if $k_{n}\left(\xi^{*}\right)=k_{n-j}\left(\xi^{*}\right)$ and $\gamma_{n-j}<\frac{1}{2}$ if and only if $k_{n}\left(\xi^{*}\right)<$ $k_{n-j}\left(\xi^{*}\right)(n-j \in I)$. Consequently we have for the set $\mathscr{M}\left(\xi^{*}\right)$ in Theorem 2.1 and the canonical moments defined in (2.6)

$$
\begin{gathered}
n \in \mathscr{M}\left(\xi^{*}\right)=\left\{j \in I \mid \gamma_{j} \geqq \frac{1}{2}\right\} \\
p_{2 j}^{*}=\frac{1}{2} \quad \text { if } j \notin \mathscr{M}\left(\xi^{*}\right) .
\end{gathered}
$$

In the following let $P_{l}\left(x, \xi^{*}\right)$ denote the lth monic orthogonal polynomial with respect to the measure $d \xi^{*}(x)$ and define

$$
\alpha_{j}=\prod_{i=1}^{j-1} \frac{q_{2 i}^{*}}{p_{2 i}^{*}}\left(1-\frac{q_{2 j}^{*}}{p_{2 j}^{*}}\right)
$$

which have sum 1 and are nonnegative, by the definition of $p_{2 j}^{*}$ in (2.6). From Theorem 3.5 and 4.1 in Dette [5] it follows that the orthogonal polynomials $k_{l}^{-1 / 2}\left(\xi^{*}\right) P_{l}\left(x, \xi^{*}\right)$ with respect to the measure $d \xi^{*}(x)$ satisfy

$$
\sum_{j=1}^{n} \alpha_{j} k_{j}^{-1}\left(\xi^{*}\right) P_{j}^{2}\left(x, \xi^{*}\right)=\sum_{j \in \mathcal{M}\left(\xi^{*}\right)} \alpha_{j} k_{j}^{-1}\left(\xi^{*}\right) P_{j}^{2}\left(x, \xi^{*}\right) \leqq 1
$$

for all $x \in[-b, b]$. Note that the result in Dette [5] was originally stated on the interval $[-1,1]$ but can easily be transferred to the interval $[-b, b]$ and that we have used $\alpha_{j} \geqq 0, \alpha_{j}=0$ if $j \notin \mathscr{M}\left(\xi^{*}\right)$, which follows from (2.8) and (2.9). By (2.10) we thus have

$$
\left\{P_{j}^{*}(x)\right\}_{j \in I}:=\left\{\sqrt{\frac{\alpha_{j}}{k_{j}\left(\xi^{*}\right)}} P_{j}\left(x, \xi^{*}\right)\right\}_{j \in I} \in P_{I}
$$

and using the definition of $\mathscr{M}\left(\xi^{*}\right)$ and $\sum_{j \in \mathscr{M}\left(\xi^{*}\right)} \alpha_{j}=1$ we obtain

$$
\sum_{j=1} m_{j}^{2}\left(P_{j}^{*}\right)=\sum_{j \in \mathcal{M}\left(\xi^{*}\right)} m_{j}^{2}\left(P_{j}^{*}\right)=\frac{1}{k_{n}\left(\xi^{*}\right)}=\max \left\{k_{j}^{-1}\left(\xi^{*}\right) \mid j \in I\right\}
$$


Therefore we have equality in Theorem 2.1 for $\left\{P_{j}^{*}\right\}_{j \in I} \in P_{I}$ and $\xi^{*} \in \Xi$ and the assertion of the theorem follows.

Remark 2.3. In the statistical theory the support points and the weights of the optimal probability measure (minimizing $\left(\mathscr{D}_{I}\right)$ ) give the relative frequencies and locations of the observations in a polynomial regression. For the special index set $I=\{1, \ldots, n\}$ this measure has been determined explicitly in [3].

Remark 2.4. The polynomial $P_{j}^{*}$ in the set $\left\{P_{j}^{*}\right\}_{j \in I}$ vanishes, whenever $j \notin \mathscr{M}\left(\xi^{*}\right)$ (which follows from $p_{2 j}^{*}=\frac{1}{2}$ and (2.9)), however, there might be situations where $\alpha_{j}=0$ also for some $j \in \mathscr{M}\left(\xi^{*}\right)$. Observing the arguments at the end of the proof of the preceding theorem the solution of $\left(\mathscr{P}_{I}\right)$ is obtained from (2.11) where the monic polynomials (orthogonal with respect to the measure $d \xi^{*}(x)$ ) are given by $(2.4)$ and the quantities $k_{j}\left(\xi^{*}\right)$ are obtained from (2.5). This provides a complete solution of the extremal problem $\left(\mathscr{P}_{I}\right)$. In the following we will discuss some special cases of the set $I$ for which this solution becomes more transparent.

\section{Chebyshev polynomials of the first kind}

If $I=\{n\}$, the solution of $\left(\mathscr{P}_{I}\right)$ is given by the Chebyshev polynomial of the first kind (on the interval $[-b, b]$ ) $T_{n}\left(\frac{x}{b}\right)$ (see Rivlin [10] or Natanson [7]). In this section we will discuss two other sets for which the extremal polynomials have a relative simple structure, namely $I=\{1, \ldots, n\}$ and $I=\{n-1, n\}$. It turns out that the answer of the question if the Chebyshev polynomial of the first kind is also a solution of $\left(\mathscr{P}_{I}\right)$ for these sets will depend heavily on the length of the interval $[-b, b]$. We will start with the discussion of the problem $\left(\mathscr{P}_{I}\right)$ for the set $I=\{1,2, \ldots, n\}$. In the following $U_{n}(x)$ denotes the Chebyshev polynomial of the second kind (on the interval $[-1,1]$ ).

Theorem 3.1. Let $I=\{1, \ldots, n\}$ and

$$
k=\min \left\{j \in\{1, \ldots, n\} \mid U_{2 n-2 i+1}\left(\frac{b}{2}\right)>0 \quad \text { for } i=j, \ldots, n\right\}
$$

then the solution of the extremal problem $\left(\mathscr{P}_{I}\right)$ is given by the polynomials $\left\{P_{l}^{*}(x)\right\}_{i=1}^{n}$ where $P_{l}^{*}(x)=0$ if $l \leqq k-1$,

$$
P_{l}^{*}(x)=\beta_{l}\left[T_{k}\left(\frac{x}{b}\right) U_{l-k}\left(\frac{x}{2}\right)-\frac{U_{n-k+1}\left(\frac{b}{2}\right)}{U_{n-k}\left(\frac{b}{2}\right)} T_{k-1}\left(\frac{x}{b}\right) U_{l-1-k}\left(\frac{x}{2}\right)\right]
$$

$(l=k, \ldots, n)$ and

$$
\beta_{1}= \pm \frac{\sqrt{b U_{2 n-21+1}\left(\frac{b}{2}\right)}}{U_{n-k+1}\left(\frac{b}{2}\right)} \quad(l=k, \ldots, n)
$$

The maximum value of $\left(\mathscr{P}_{I}\right)$ is given by 


$$
\frac{2^{2 k-2}}{b^{2 k-1}} \frac{U_{n-k}\left(\frac{b}{2}\right)}{U_{n-k+1}\left(\frac{b}{2}\right)}
$$

Proof. For $j=n, \ldots, k$ define $\gamma_{j}\left(\xi^{*}\right)=1-b^{-2(n-j)} \prod_{i=j+1}^{n-1}\left(q_{2 i}^{*} p_{2 i}^{*}\right)^{-1}$ (here we put $\gamma_{n}\left(\xi^{*}\right)=1$ and the $p_{2 j}^{*}$ are defined by (2.6)), then it is straighforward to show that

$$
\gamma_{j}\left(\xi^{*}\right)=\frac{U_{n-j+1}\left(\frac{b}{2}\right)}{b U_{n-j}\left(\frac{b}{2}\right)}=\frac{U_{2 n-2 j+1}\left(\frac{b}{2}\right)}{2 b U_{n-j}^{2}\left(\frac{b}{2}\right)}+\frac{1}{2} \quad j=k, \ldots, n
$$

and the definition of $k$ in (3.1) and Theorem 2.2 yield for the canonical moments of the solution $\xi^{*}$ of the dual problem $\left(\mathscr{D}_{I}\right) p_{2 j}^{*}=\gamma_{j}\left(\xi^{*}\right)(j=k, \ldots, n)$. If $k \geqq 2$, then it follows that

$$
\gamma_{k-1}\left(\xi^{*}\right)=1-b^{-2(n-k+1)} \prod_{i=k}^{n-1}\left(q_{2 i}^{*} p_{2 i}^{*}\right)^{-1} \leqq \frac{1}{2}
$$

and that $b \leqq 2$ which implies (by Theorem 2.2) $p_{2 k-2}^{*}=\frac{1}{2}$ and $\gamma_{k-2}\left(\xi^{*}\right) \leqq 1-2 b^{-2} \leqq \frac{1}{2}$. Therefore the canonical moments of the solution $\xi^{*}$ of the dual problem $\left(\mathscr{D}_{1}\right)$ in Theorem 2.2 are given by

$$
\left(\frac{1}{2}, \frac{1}{2}, \ldots, \frac{1}{2}, p_{2 k}^{*}, \frac{1}{2}, p_{2 k+2}^{*}, \frac{1}{2}, \ldots, \frac{1}{2}, p_{2 n-2}^{*}, \frac{1}{2}, 1\right)
$$

where $p_{2 j}^{*}=\gamma_{j}\left(\xi^{*}\right)(j=k, \ldots, n)$ and $\gamma_{j}\left(\xi^{*}\right)$ is defined in (3.3). By Theorem 2.1 we have to find the orthonormal polynomials with respect to the measure $d \xi^{*}(x)$ whose monic form is given by (2.4) that is

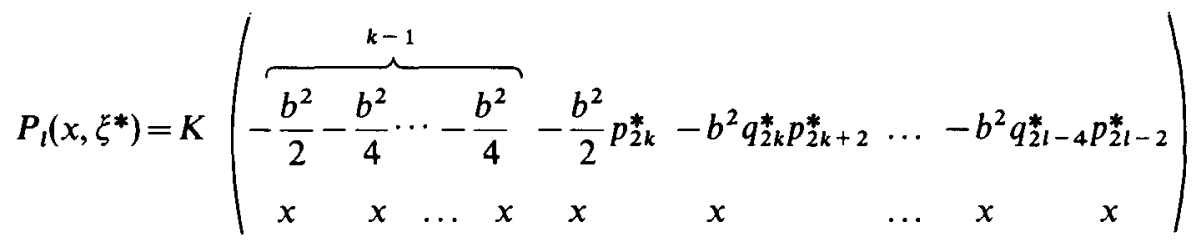

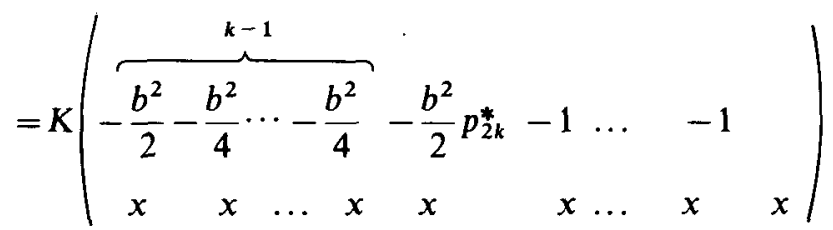

$$
\begin{aligned}
& =2^{-k+1} b^{k}\left[T_{k}\left(\frac{x}{b}\right) U_{l-k}\left(\frac{x}{2}\right)-\frac{U_{n-k+1}\left(\frac{b}{2}\right)}{U_{n-k}\left(\frac{b}{2}\right)} T_{k-1}\left(\frac{x}{b}\right) U_{l-1-k}\left(\frac{x}{2}\right)\right]
\end{aligned}
$$

Here we used Sylvester's identity (see e.g. Studden [11, formula (4.12)]), (3.3) (for $j=k$ ) and the recursive definition of the Chebyshev polynomials of the first and second kind. 
Note that the case $k=1$ has to be considered separately but gives the corresponding result in (3.4) for $k=1$. The $L_{2}$-norm of this polynomial is given by (note that $p_{2 j}^{*}=\frac{1}{2}, j=1, \ldots, k-1$ and $\left.p_{2 j}^{*}=\gamma_{j}\left(\xi^{*}\right), j=k, \ldots, n\right)$

$$
k_{l}\left(\xi^{*}\right)=b^{2 l}\left(\frac{1}{4}\right)^{k-1} p_{2 k}^{*} \prod_{j=k+1}^{l} q_{2 j-2}^{*} p_{2 j}^{*}=\frac{b^{2 k-1}}{2^{2 k-2}} \frac{U_{n-k+1}\left(\frac{b}{2}\right)}{U_{n-k}\left(\frac{b}{2}\right)}
$$

while the quantities $\alpha_{l}$ in (2.9) are obtained as

$$
\alpha_{1}=\frac{U_{n-l}\left(\frac{b}{2}\right)\left[U_{n-l+1}\left(\frac{b}{2}\right)-U_{n-l-1}\left(\frac{b}{2}\right)\right]}{U_{n-k}\left(\frac{b}{2}\right) U_{n-k+1}\left(\frac{b}{2}\right)}=\frac{U_{2 n-2 l+1}\left(\frac{b}{2}\right)}{U_{n-k}\left(\frac{b}{2}\right) U_{n-k+1}\left(\frac{b}{2}\right)}
$$

$(l=k, \ldots, n)$. The assertion now follows from Theorem 2.1 .

3.2 Discussion. Theorem 3.1 shows that the structure of the solution of $\left(\mathscr{P}_{l}\right)$ changes completely with the length of the interval $[-b, b]$. If $b \leqq \sqrt{2}$, then we obtain from (3.1) $k=n$ and consequently the sum of the squared leading coefficients of the polynomials $P_{1}^{*}, \ldots, P_{n}^{*}$ is maximized for the choice $P_{l}^{*}(x)=0(1 \leqq l \leqq n-1)$ and $P_{n}^{*}(x)=T_{n}\left(\frac{x}{b}\right)$ with maximum value $\left(2^{n-1} b^{-n}\right){ }^{2}$ If $b>\sqrt{2}$ the situation changes completely. In this case the index $1 \leqq k \leqq n$ defined by (3.1) depends on $n$ and $b$. The solution of the problem $\left(\mathscr{P}_{I}\right)$ is given by (3.2). Finally, if $b \geqq 2$, it follows that $k=1$ and (3.2) simplifies to

$$
P_{l}^{*}(x)=\frac{\sqrt{U_{2 n-2 l+1}\left(\frac{b}{2}\right)}}{\sqrt{b} U_{n}\left(\frac{b}{2}\right)}\left[U_{l}\left(\frac{x}{2}\right)-\frac{U_{n+1}\left(\frac{b}{2}\right)}{U_{n-1}\left(\frac{b}{2}\right)} U_{l-2}\left(\frac{x}{2}\right)\right] \quad l=1, \ldots, n
$$

Example 3.3. Let $n=3$, then we have to distinguish the following cases:

(A) If $b \leqq \sqrt{2}$, we have $k=3$, the optimal polynomials are given by

$$
P_{1}^{*}(x)=P_{2}^{*}(x)=0, \quad P_{3}^{*}(x)= \pm T_{3}\left(\frac{x}{b}\right)
$$

and the maximum is $16 b^{-6}$.

(B) If $\sqrt{2} \leqq b \leqq \sqrt{3}$, then $k=2$, the optimal polynomials are

$$
\begin{aligned}
& P_{1}^{*}(x)=0, \quad P_{2}^{*}(x)= \pm \frac{b \sqrt{b^{2}-2}}{b^{2}-1} T_{2}\left(\frac{x}{b}\right) \\
& P_{3}^{*}(x)= \pm \frac{b}{b^{2}-1}\left[x T_{2}\left(\frac{x}{b}\right)-\frac{b^{2}-1}{b} T_{1}\left(\frac{x}{b}\right)\right]= \pm \frac{1}{2\left(b^{2}-1\right)}\left[b^{2} T_{3}\left(\frac{x}{b}\right)-\left(b^{2}-2\right) T_{1}\left(\frac{x}{b}\right)\right]
\end{aligned}
$$


and the maximum value is $4 b^{-2}\left(b^{2}-1\right)^{-1}$.

(C) If $b \geqq \sqrt{3}$, then $k=1$, the optimal polynomials are

$$
\begin{aligned}
& P_{1}^{*}(x)= \pm \frac{\sqrt{b^{4}-4 b^{2}+3}}{b^{3}-2 b} x, \quad P_{2}^{*}(x)= \pm \frac{1}{b \sqrt{b^{2}-2}}\left[U_{2}\left(\frac{x}{2}\right)-\frac{b^{4}-3 b^{2}+1}{b^{2}-1}\right] \\
& P_{3}^{*}(x)= \pm \frac{1}{b^{3}-2 b}\left[U_{3}\left(\frac{x}{2}\right)-\frac{b^{4}-3 b^{2}+1}{b^{2}-1} U_{1}\left(\frac{x}{2}\right)\right]
\end{aligned}
$$

and the maximum value is $\left(b^{2}-1\right) /\left[b^{2}\left(b^{2}-2\right)\right]$.

In the remaining part of this section we will consider the index set $I=\{n-1, n\}$. Thus the problem is to maximize the sum of the squared coefficients

$$
m_{n-1}^{2}\left(P_{n-1}\right)+m_{n}^{2}\left(P_{n}\right)
$$

over the set of all polynomials (of degree $n-1$ and $n$ ) satisfying

$$
P_{n-1}^{2}(x)+P_{n}^{2}(x) \leqq 1 \text { for all } x \in[-b, b] .
$$

The solution of this problem can be obtained by a similar reasoning as in Theorem 3.1 for $k=n$ and $k=n-1$ and we omit the details in the proof of the following result.

Theorem 3.4. The polynomials $P_{n-1}^{*}(x)$ and $P_{n}^{*}(x)$ maximizing (3.4) subject to the restriction (3.5) are given by

$$
\begin{aligned}
& \left(P_{n-1}^{*}(x), P_{n}^{*}(x)\right)=\left(0, \pm T_{n}\left(\frac{x}{b}\right)\right) \text { if } b \leqq \sqrt{2} \\
& \left(P_{n-1}^{*}(x), P_{n}^{*}(x)\right)=\left( \pm \frac{b \sqrt{b^{2}-2}}{b^{2}-1} T_{n-1}\left(\frac{x}{b}\right), \pm \frac{1}{2\left(b^{2}-1\right)}\left[b^{2} T_{n}\left(\frac{x}{b}\right)-\left(b^{2}-2\right) T_{n-2}\left(\frac{x}{b}\right)\right]\right)
\end{aligned}
$$

if $b \geqq \sqrt{2}$. The maximum values in (3.4) are given by $2^{2 n-2} b^{-2 n}$, if $b \leqq \sqrt{2}$, and by $2^{2 n-4} b^{-(2 n-4)}\left(b^{2}-1\right)^{-1}$ if $b \geqq \sqrt{2}$, respectively.

Remark 3.5. For index sets of the form $I_{m}=\{n-m+1, \ldots, n\}$ the corresponding results are obtained similar to Theorem 3.4. The values of $b$ where the structure of the 
solution is changing, are obtained successively from (3.1) as $b=\sqrt{2}, b=\sqrt{3}, b=$ $\sqrt{2+\sqrt{2}}, b=(\sqrt{5+\sqrt{5}}) / \sqrt{2}, \ldots$ (see also Example 3.3).

\section{Chebyshev polynomials of the second kind}

In this section we will briefly discuss some generalizations of the extremal properties of the Chebyshev polynomials of the second kind. Let $I$ denote a subset of $\{0,1, \ldots, n\}$ and define

$$
\tilde{P}_{I}:=\left\{\left(P_{j}\right)_{j \in I} \mid P_{j} \in \mathscr{P}_{j}, j \in I, \sup _{x \in[-b, b]}\left(b^{2}-x^{2}\right) \sum_{j \in I} P_{j}^{2}(x) \leqq 1\right\}
$$

as the set of all polynomials $\left(P_{j}\right)_{j \in I}$ such that a weighted sup-norm of the sum of squares is less or equal 1 . We are interested in the problem

$$
\max \left\{\sum_{l \in I} m_{l}^{2}\left(P_{l}\right) \mid\left(P_{l}\right)_{l \in I} \in \tilde{P}_{I}\right\}
$$

If $I=\{n\}$ we obtain the well known extremal property of the Chebyshev polynomials of the second kind $U_{n}(x)$, if $b=1$, (see e.g. Achieser [1, p. 250]) and more generally of $U_{n}\left(\frac{x}{b}\right) / b$, if $b>0$. For the sake of brevity we will only state the generalizations corresponding to the index sets $I=\{0, \ldots, n\}$ and $I=\{n-1, n\}$. All proofs can be obtained by a similar reasoning as in the previous sections and are therefore omitted.

Theorem 4.1. Let $I=\{0, \ldots, n\}$ and

$$
k=\min \left\{j \in\{0, \ldots, n+1\} \mid U_{2 n-2 i+3}\left(\frac{b}{2}\right)>0 \quad \text { for } i=j, \ldots, n+1\right\}
$$

then the solution of the problem $\left(\mathscr{P}_{l}\right)$ is given by the polynomials $\left\{P_{l}^{*}(x)\right\}_{l=0}^{n}$ where $P_{l}^{*}(x)=0$ if $l \leqq k-2$ and

$$
P_{l}^{*}(x)=\tilde{\beta}_{l}\left[U_{k-1}\left(\frac{x}{b}\right) U_{l-k+1}\left(\frac{x}{2}\right)-\frac{U_{n-k+2}\left(\frac{b}{2}\right)}{U_{n-k+1}\left(\frac{b}{2}\right)} U_{k-2}\left(\frac{x}{b}\right) U_{l-k}\left(\frac{x}{2}\right)\right]
$$

$(l=k-1, \ldots, n)$, where

$$
\widetilde{\beta}_{l}= \pm \frac{\sqrt{U_{2 n-2 l+1}\left(\frac{b}{2}\right)}}{\sqrt{b} U_{n-k+2\left(\frac{b}{2}\right)}} \quad(l=k-1, \ldots, n) .
$$

The maximum value of $\left(\mathscr{P}_{1}\right)$ is given by 


$$
\frac{2^{2 k-2}}{b^{2 k-1}} \frac{U_{n-k+1}\left(\frac{b}{2}\right)}{U_{n-k+2}\left(\frac{b}{2}\right)}
$$

Remark 4.2. If $b \leqq \sqrt{2}$ then it follows from (4.1) that $k=n+1$ and the solution of $\left(\mathscr{P}_{l}\right)$ is given by the polynomials $P_{l}^{*}(x)=0, l=0, \ldots, n-1$, and $P_{n}^{*}(x)=\frac{1}{b} U_{n}\left(\frac{x}{b}\right)$. As in Discussion 3.2 it follows that for $b \geqq 2$ we have $k=1$ and the optimal polynomials are "essentially" independent of the interval $[-b, b]$ and proportional to the Chebyshev polynomials of the second kind, that is

$$
P_{l}^{*}(x)= \pm \frac{\sqrt{U_{2 n-2 l+1}\left(\frac{b}{2}\right)}}{\sqrt{b} U_{n-1}\left(\frac{b}{2}\right)} U_{l}\left(\frac{x}{2}\right) \quad(l=0,1, \ldots, n)
$$

with maximum value $U_{n}\left(\frac{b}{2}\right)\left[b U_{n+1}\left(\frac{b}{2}\right)\right]^{-1}$. In the interval $[\sqrt{2}, 2]$ we have $1 \leqq k \leqq n+1$ (depending on $b$ and $n$ ) and the solution of $\left(\mathscr{P}_{I}\right)$ is given by (4.1) and (4.2).

Theorem 4.3. Let $b \leqq \sqrt{2}$, then the solution of the problem

$$
\text { maximize } m_{n-1}^{2}\left(P_{n-1}\right)+m_{n}^{2}\left(P_{n}\right)
$$

subject to the restriction

$$
\sup _{x \in[-b, b]}\left(b^{2}-x^{2}\right)\left[P_{n-1}^{2}(x)+P_{n}^{2}(x)\right] \leqq 1
$$

is given by the polynomials $P_{n-1}^{*}(x)=0, P_{n}^{*}(x)=\frac{1}{b} U_{n}\left(\frac{x}{b}\right)$ with optimum value $2^{2 n} b^{-2 n+2}$. If $b \geqq \sqrt{2}$ the maximum in (4.3) subject to (4.4) is attained for the polynomials

$$
\left(P_{n-1}^{*}(x), P_{n}^{*}(x)\right)=\left( \pm \frac{\sqrt{b^{2}-2}}{b^{2}-1} U_{n-1}\left(\frac{x}{b}\right), \pm \frac{b}{2\left(b^{2}-1\right)}\left[U_{n}\left(\frac{x}{b}\right)-\frac{\left(b^{2}-2\right)}{b^{2}} U_{n-2}\left(\frac{x}{b}\right)\right]\right)
$$

with maximum value $(2 / b)^{2(n-1)}\left(b^{2}-1\right)^{-1}$.

\section{Appendix}

(Proof of Theorem 2.1) The proof of Theorem 2.1 follows from a standard result in the theory of optimal design in mathematical statistics (see Pukelsheim [9]). To be precise let $I=\left\{i_{1}, \ldots, i_{m}\right\}, i=m+\sum_{j=1}^{m} i_{j}, f_{j}(x)=\left(1, x, \ldots, x^{j}\right)^{\prime}$ (where ' denotes transposition) and define for a probability measure $\xi$ on the interval $[-b, b]$

$$
M_{j}(\xi)=\int_{-b}^{b} f_{j}(x) f_{j}(x)^{\prime} d \xi(x) \in \mathbb{R}^{(j+1) \times(j+1)} \quad(j \in I)
$$


which is called moment matrix in the theory of optimal design. In the following we will collect all matrices $M_{i_{1}}(\xi), \ldots, M_{i_{m}}(\xi)$ in one big matrix

$$
M(\xi)=\left(\begin{array}{ccc}
M_{i_{1}}(\xi) & & \\
& \ddots & \\
& & M_{i_{m}}(\xi)
\end{array}\right) \in \mathbb{R}^{i \times i}
$$

and define two matrices by

$$
K=\left(\begin{array}{ccc}
e_{i_{1}} & & \\
& \ddots & \\
& & e_{i_{m}}
\end{array}\right) \in \mathbb{R}^{i \times m} \quad N=\left(\begin{array}{ccc}
N_{i_{1}} & & \\
& \ddots & \\
& & N_{i_{m}}
\end{array}\right) \in \mathbb{R}^{i \times i}
$$

where $e_{j}=(0, \ldots, 0,1)^{\prime} \in \mathbb{R}^{j+1}$ is the $(j+1)$ th unit vector $(j \in I), N_{i j}$ are nonnegative $\left(i_{j}+1\right) \times\left(i_{j}+1\right)$ matrices (i.e. $N_{i_{j}} \geqq 0$ ) and all other entries in these matrices are 0 . Defining $\Phi_{-\infty}(A)=\lambda_{\text {min }}(A)$ where $A \in \mathbb{R}^{m \times m}, A \geqq 0$ and $\lambda_{\text {min }}(A)$ denotes the minimum eigenvalue of $A$ we obtain for the polar function of $\Phi_{-\infty}$ (see Pukelsheim [9, p. 149]) $\Phi_{-\infty}^{\infty}(A)=\operatorname{trace}(A)$. By the duality theorem on page 172 in the same reference it now follows that (note that $k_{j}(\xi)=\left[e_{j}^{\prime} M_{j}^{-1}(\xi) e_{j}\right]^{-1}$ )

$$
\begin{aligned}
& \max _{\xi \in \Xi} \min \left\{k_{j}(\xi) \mid j \in I\right\}=\max _{\xi \in \Xi} \Phi_{-\infty}\left(\left(K^{\prime} M^{-1}(\xi) K\right)^{-1}\right) \\
& \quad=\min \left\{\left[\Phi_{-\infty}^{\infty}\left(K^{\prime} N K\right)\right]^{-1} \mid N \in \mathbb{R}^{i \times i}, N \geqq 0, \operatorname{trace}(M(\xi) N) \leqq 1 \forall \xi \in \Xi\right\} \\
& \quad=\min \left\{\left(\sum_{j \in I} e_{j}^{\prime} N_{j} e_{j}\right)^{-1} \mid N_{j} \in \mathbb{R}^{(j+1) \times(j+1)}, N_{j} \geqq 0 \forall j \in I, \sum_{j \in I} \operatorname{trace}\left(M_{j}(\xi) N_{j}\right) \leqq 1 \forall \xi \in \Xi\right\}(\mathrm{A}) \\
& =\min \left\{\left(\sum_{j \in I}\left(e_{j}^{\prime} a_{j}\right)^{2}\right)^{-1} \mid a_{j} \in \mathbb{R}^{j+1} \forall j \in I, \sum_{j \in I}\left(f_{j}(x)^{\prime} a_{j}\right)^{2} \leqq 1 \forall x \in[-b, b]\right\} \\
& \quad=\min \left\{\left(\sum_{j \in I} m_{j}^{2}\left(P_{j}\right)\right)^{-1} \mid\left(P_{j}\right)_{j \in I} \in P_{I}\right\} .
\end{aligned}
$$

In order to go from the third to the fourth line in (A1) we have used that

$$
\sum_{j \in I} \operatorname{trace}\left(M_{j}(\xi) N_{j}\right)=\sum_{j \in I} \int_{-b}^{b} f_{j}(x)^{\prime} N_{j} f_{j}(x) d \xi(x) \leqq 1 \quad \forall \xi \in \Xi
$$

is equivalent to the inequality

$$
\sum_{j \in I} f_{j}(x)^{\prime} N_{j} f_{j}(x) \leqq 1 \quad \forall x \in[-b, b]
$$


and the fact that the minimum value does not change if the matrices $N_{j}$ are replaced by matrices of the form $a_{j} a_{j}^{\prime}$ (see the following discussion). This proves the first part of the Theorem. For the second part we discuss equality in (A1) that is equality in the duality theorem in Pukelsheim [9, p. 171, 172]) and obtain

$$
\begin{gathered}
\sum_{j \in I} \operatorname{trace}\left(M_{j}\left(\xi^{*}\right) N_{j}\right)=1 \\
M_{j}\left(\xi^{*}\right) N_{j}=\frac{e_{j} e_{j}^{\prime} N_{j}}{e_{j}^{\prime} M_{j}^{-1}\left(\xi^{*}\right) e_{j}} j \in I \\
\min _{j \in I}\left\{\left(e_{j}^{\prime} M_{j}^{-1}\left(\xi^{*}\right) e_{j}\right)^{-1}\right\} \sum_{j \in I} e_{j}^{\prime} N_{j} e_{j}=\sum_{j \in I} \frac{e_{j}^{\prime} N_{j} e_{j}}{e_{j}^{\prime} M_{j}^{-1}\left(\xi^{*}\right) e_{j}}=1 .
\end{gathered}
$$

Observing that $k_{j}^{-1}\left(\xi^{*}\right)=e_{j}^{\prime} M_{j}^{-1}\left(\xi^{*}\right) e_{j}(j=1, \ldots, n)$ we obtain by straightforward calculation as a solution of (A3) and (A4) $N_{j}=\alpha_{j} a_{j} a_{j}^{\prime}$ where $a_{j}=\sqrt{k_{j}\left(\xi^{*}\right)} M_{j}^{-1}\left(\xi^{*}\right) e_{j}(j \in I), \alpha_{j} \geqq 0$ (because $N_{j} \geqq 0$ ) and $\sum_{j \in I} \alpha_{j}=1$. Finally it follows from (A5) that $\alpha_{j}=0$ whenever $j \notin \mathscr{M}\left(\xi^{*}\right)$. By Corollary 2.3 in Dette [5] the polynomials $P_{l}^{*}\left(x, \xi^{*}\right)=a_{l}^{\prime} f_{l}(x)$ are orthonormal with respect to the measure $d \xi^{*}(x)$ which yield for the monic orthogonal polynomials $P_{l}\left(x, \xi^{*}\right)=\sqrt{k_{l}\left(\xi^{*}\right)} a_{l}^{\prime} f_{l}(x)(l=1, \ldots, n)$. Consequently a solution of the right hand side of $(\mathrm{A} 1)$ is given by $\left\{\sqrt{\alpha_{j} / k_{j}\left(\xi^{*}\right)} P_{j}\left(x, \xi^{*}\right)\right\}_{j \in I}$ where $P_{j}\left(x, \xi^{*}\right)$ is the $j$ th monic orthogonal polynomial with respect to the measure $d \xi^{*}(x)$ and the $\alpha_{j}$ have to satisfy

$$
\sum_{j \in I} \alpha_{j} k_{j}^{-1}\left(\xi^{*}\right) P_{j}^{2}\left(x, \xi^{*}\right)=\sum_{j \in I} f_{j}(x)^{\prime} N_{j} f_{j}(x) \leqq 1
$$

for all $x \in[-b, b]$. This completes the proof of Theorem 2.1.

\section{REFERENCES}

1. N. I. ACHIESER, Theory of Approximation (Dover, New York, 1956).

2. T. S. Chimara, An Introduction to Orthogonal Polynomials (Gordon and Breach, New York, 1978).

3. H. Dette, Optimal designs for identifying the degree of a polynomial regression, Ann. Statist. (1995), to appear.

4. H. DeTre, Extremal properties for ultraspherical polynomials, J. Approx. Theory 76 (1994), 246-273.

5. H. Detre, New identities for orthogonal polynomials on a compact interval, J. Math. Anal. Appl. 179 (1994), 547-573.

6. T. S. LaU W. J. Studden, On an extremal problem of Fejér, J. Approx. Theory 53 (1988), 184-194.

7. I. P. Natanson, Konstruktive Funktionentheorie (Akademie Verlag, Berlin, 1955).

8. O. Perron, Die Lehre von den Kettenbrüchen (Band I, II) (B. G. Teubner, Stuttgart, 1954).

9. F. Pukelsheim, Optimal Design of Experiments (Wiley, New York, 1993). 
10. T. J. Rivun, Chebyshev polynomials (Wiley, New York, 1990).

11. W. J. StudDen, $D_{s}$-optimal designs for polynomial regression using continued fractions, Ann. Statist. 8 (1980), 1132-1141.

12. W. J. Studden, On a problem of Chebyshev, J. Approx. Theory 29 (1981), 253-260.

13. H. S. WALl, Analytic theory of continued fractions (Van Nostrand, New York, 1948).

InStITUt for Mathematische Stochastik

Abteilung Mathematik

TeChNISChe UnIVERSITÄt DresDen

MOMMSENSTR, 13

01062 DRESDEN

Germany 